\title{
Study of Group Food Retrieval by Ants as a Model for Multi-robot Collective Transport Strategies
}

\author{
Spring Berman*1, Quentin Lindsey ${ }^{\dagger}$, Mahmut Selman Sakar ${ }^{\ddagger 1}$, Vijay Kumar ${ }^{\dagger}$, and Stephen Pratt ${ }^{\S}$ \\ * School of Engineering and Applied Sciences, Harvard University, Cambridge, MA 02138 USA \\ ${ }^{\dagger}$ GRASP Laboratory, University of Pennsylvania, Philadelphia, PA 19104 USA \\ $\ddagger$ Department of Mechanical Engineering, Massachusetts Institute of Technology, Cambridge, MA 02139 USA \\ ${ }^{\S}$ School of Life Sciences, Arizona State University, Tempe, AZ 85287 USA
}

\begin{abstract}
Group food retrieval in some ant species serves as a useful paradigm for multi-robot collective transport strategies that are decentralized, scalable, and do not require a priori information about the payload. We investigate this phenomenon in Aphaenogaster cockerelli in order to extract the ants' roles during transport, the rules that govern their actions, and the individual forces that they apply to guide a food item to their nest. To measure these forces, we designed and fabricated elastic structures with calibrated stiffness properties, induced ants to retrieve the structures, and tracked the resulting deformations with a camera. We then developed a hybrid system model of the ant behaviors that were observed in the experiments. We conducted simulations of the behavioral model that incorporate a quasistatic model of planar manipulation with compliant attachment points. Our simulations qualitatively replicate individual ant activity as well as certain macroscopic features of the transport.
\end{abstract}

\section{INTRODUCTION}

While there are various approaches to cooperative robotic manipulation, there are few decentralized approaches that are applicable to large groups of mobile robots. Such approaches can provide a greater degree of flexibility and robustness in construction and manufacturing applications. Cooperative food retrieval in ants is a striking example of a fully decentralized manipulation strategy that is scalable in the number of transporters and successful for a wide range of loads and environments. This biological phenomenon offers inspiration for the analogous problem in robotics: a group of robots is tasked to manipulate an arbitrarily-shaped payload, which is too heavy for a single robot to move, to a target destination without a priori knowledge about the payload or obstacles in the environment. The robots must rely on local sensing and no explicit communication in order for the strategy to be scalable.

To extract the rules that govern ant transport behavior and better understand the mechanisms of successful collective transport, we study this phenomenon in Aphaenogaster cockerelli (Fig. 1), a monomorphic ant species that is common in the deserts of the southwestern United States. To the authors' knowledge, this is the first work that investigates the mechanics of cooperative transport in ants. We fabricate elastic twodimensional structures that we use as vision-based ant force sensors and videotape ant retrieval of the structures. Using

${ }^{1}$ This work was done when Spring Berman (email: sberman@eecs.harvard.edu) and Mahmut Selman Sakar were affiliated with the GRASP Laboratory at the University of Pennsylvania.

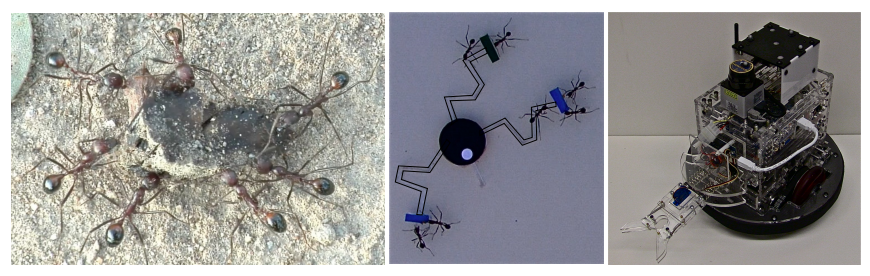

Fig. 1. From left to right: $A$. cockerelli ants retrieving a piece of fig and an elastic structure (springs are outlined for clarity), described in Section III-A, and a SCARAB differential-drive robot [15] equipped with a single-actuator gripper with passive compliance.

the video data, we quantitatively illustrate salient features of ant transport, including the emergence of consensus. We then develop a dynamic and behavioral model of ant transport in a step toward implementation on robotic platforms such as the one in Fig. 1. We validate the model by reproducing the observed characteristics of transport through simulations.

\section{LITERATURE REVIEW}

\section{A. Collective transport in ants}

Group retrieval has evolved independently many times in the ants, but it is developed to much higher levels in some species than in others [6]. Groups can lift more weight per worker than solitary carriers, enhancing the colony's efficiency at food collection. The small colonies of $A$. cockerelli are skillful at group retrieval [5]. When a solitary forager finds an item too large for her to move, she summons a team of nestmates within a few minutes. They quickly make off with the food, pre-empting competitors whose mass recruitment abilities and greater aggression would otherwise allow them to displace the relatively timid $A$. cockerelli.

Collective transport is a stigmergic process in the sense that the medium of communication between ants is the progress of the transport itself [22]. The tractive resistance of the prey stimulates orientational and positional changes by the ants and recruitment behavior [21], [23]. Transport has been observed to consist of an "uncoordinated" phase in which ants arrive at the prey, either by chance or via recruitment, and pull on it in all directions, followed by a "coordinated" phase during which a preferred direction emerges from the ants' exertions and transport occurs [12]. The phase transition has been attributed 
to spatial rearrangements [16], a decrease in number of ants, realignments and rotation of the prey [23], and tensions acting as positive feedback when associated with success [12].

There has been some debate over the existence of cooperation among ants during transport. On one hand, ants often appear to behave as though they were isolated, at times pulling on the load in opposite directions. Indeed, they frequently exhibit the same behaviors in solitary transport and group transport [21], [23]. However, the concurrent behaviors of multiple ants during group transport produces some cooperative features: groups can move prey significantly faster [2], develop higher mean power [23], and exert greater forces than single ants [21]. The consensus is that this apparent collaboration does not arise from conscious deliberation, learning, or communication, but is rather a juxtaposition of uncoordinated individual forces on the prey with a strong component toward the nest, the common destination of the ants [12].

\section{B. Multi-robot transport systems}

Many approaches to cooperative robotic manipulation rely on centralized or leader/follower schemes that require knowledge of the load geometry and possibly the contact forces [17]. Several decentralized manipulation schemes have been developed for motion and force control of a payload by a fixed group of robots in an obstacle-free environment [8], [13], [17], [20], [24]. Groups of two robots only were used for experimental verification [8], [17], [24], and [8] requires communication among platforms. Other decentralized multirobot transport approaches include object closure and caging, which compose the robot behaviors of approach, surround, and push in order to geometrically enclose and move an object in a predictable manner [4], [19]. An ant-inspired decentralized approach to multi-robot box pushing, in which robots switch between simple behaviors in response to locally sensed cues, is presented in [9].

\section{Elastic Vision-Based Force Sensor}

\section{A. Design}

Preliminary trials were conducted with plastic disks, which were coated with fig paste to induce retrieval. A group of about 15 ants was able to transport a disk of mass $m=1.6 \mathrm{~g}$ and diameter $d=4.6 \mathrm{~cm}$. The ants were better at transport when the perimeter of the disk had small tabs, measuring $2 \mathrm{~mm}$ long and $0.5 \mathrm{~mm}$ wide, for their mandibles to grasp. More than 20 ants participated in carrying a larger disk, with $m=3.4 \mathrm{~g}$ and $d=6.9 \mathrm{~cm}$, although they were not as effective as the group carrying the smaller disk.

Using these dimensions as guidelines, we developed and tested several planar elastic structures. Each consisted of a circular load ringed with several springs that were tipped with thin tabs (see the labels on the structure in Fig. 2a). In all of the structures, some ants gripped the springs themselves rather than the tabs; however, their contributions to the spring deflections did not seem significant since most ants gripped the tabs and bars, which were smeared with fig paste. (a)

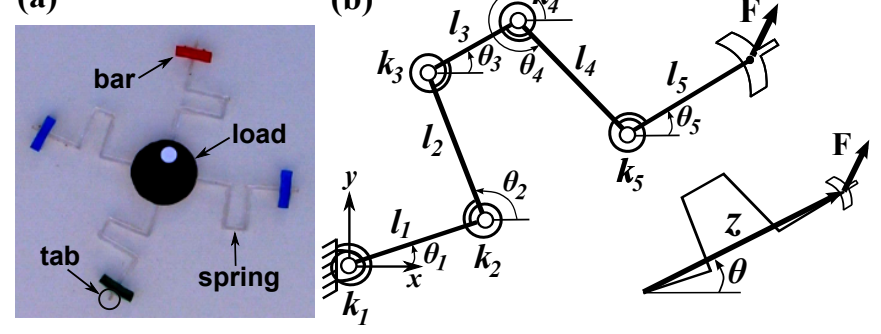

Fig. 2. (a) Elastic structure 2 with labels. (b) General pseudo-rigid-body model of a spring on the structure (see Section III-D).

The first prototype $(m=1.3 \mathrm{~g}, d=8.5 \mathrm{~cm})$ was laser cut from 0.75 -mm-thick Lexan material. A group of 12-15 ants carried the structure effectively, but they produced negligible spring deformations, even when several ants pulled on one spring. To obtain measurable deformations, we switched to fabricating 3.2-mm-thick structures out of the soft material polydimethylsiloxane, or PDMS (see Section III-B). We chose PDMS for its mechanical compliance, manufacturability, and biocompatibility [26]. When pulled by ants, a second prototype $(m=2.6 \mathrm{~g}, d=4.5 \mathrm{~cm})$ and a structure we will call structure $1(m=2.3 \mathrm{~g}, d=6.1 \mathrm{~cm})$ showed significant spring deformations in the radial and tangential directions, respectively, but not in both directions. For structure $2(m=$ $1.6 \mathrm{~g}, d=7.7 \mathrm{~cm}$ ), we modified the spring geometry, shown in Fig. 2b, to have more similar radial and tangential stiffnesses. The lengths of the spring members in $\mathrm{mm}$ are $l_{1}=9.8$, $l_{2}=l_{4}=11.9, l_{3}=5.6, l_{5}=10.9$, and the spring width is $h=0.8 \mathrm{~mm}$. Since the $\mu$ for PDMS is relatively large regardless of surface, we chose to reduce this quantity in structures 1 and 2 by applying DuPont ${ }^{\mathrm{TM}}$ Teflon $R$ tape to the bottom of the loads.

\section{B. Fabrication}

The elastic structures were fabricated using the transfer molding procedure shown in Fig. 3. The process started with the fabrication of molds of the structure shape. The substrate used for the mold was acrylic (Lucite International) because of its strength, elasticity, and ease of manufacturing. A flatbed laser cutter (Universal Laser Systems X-660) was used to cut the negative structure features (Fig. 3a). Then the mold and a $5 \mathrm{~cm} \times 5 \mathrm{~cm}$ glass plate were silanized by vapor deposition of (tridecafluoro-1,1,2,2-tetrahydrooctyl)-1-trichlorosilane for $1 \mathrm{~h}$ at room temperature in a vacuum chamber, and the mold was fixed on the glass plate.

The PDMS elastomer base (Dow Corning, MI) was polymerized by mixing 10:1 (w/w) ratio with curing agent. The solution was stirred and degassed under vacuum for $20 \mathrm{~min}$, and the mold was filled with the viscous solution. Excess solution was scraped off the surface of the mold with a clean razor blade (Fig. 3b). The polymer was cured for $2 \mathrm{~h}$ at $80^{\circ} \mathrm{C}$ on a hot plate (Fig 3c). Once cured, the structure (Fig. 2a) was carefully removed with a pair of tweezers. 


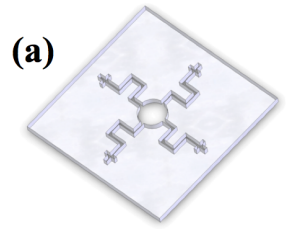

(b)

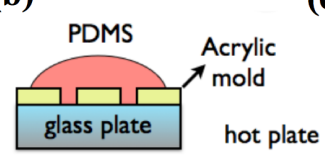

(c)

Fig. 3. Elastic structure fabrication process.

\section{Calibration}

The calibration setup uses a micro/macro manipulation apparatus to create a lookup table of applied force vs. spring tip displacement. The load of the structure is clamped to an acrylic mount such that one spring is in the appropriate configuration. This mount is attached to a motorized rotary stage (New Focus $^{\mathrm{TM}}$ ), which is mounted on a $\mathrm{H} 107$ ProScan ${ }^{\mathrm{TM}}$ II xy stage with 1 micron repeatability, step size of 5 microns, and travel of $10.16 \mathrm{~cm} \times 7.62 \mathrm{~cm}$. A needle, which is attached to a load

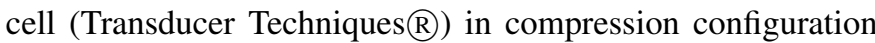
and allowed to rotate freely, is inserted into the bar at the tip of the spring. Using an automated routine, the stage moves to predetermined locations on a rectangular grid, and a force measurement is taken using the load cell at each location. To determine the radial spring force, the longitudinal axis of the spring is aligned parallel to the axis of the load cell. After the routine has swept the entire grid, the spring is returned to its rest configuration and rotated with the rotary stage $90^{\circ}$ clockwise so that its latitudinal axis is aligned with the load cell in order to measure the tangential forces. Half of the grid is swept in that configuration so that the load cell is in compression. The spring is then rotated $180^{\circ}$ counterclockwise so that the other half of the grid is swept.

\section{Model}

We develop a simple model of the springs that can be used to quickly estimate their radial and tangential stiffnesses and to design a spring geometry with desired stiffness properties. Since the springs consist of flexible members that can undergo large deflections, we apply a pseudo-rigid-body model, which describes the behavior of compliant mechanisms [7].

Fig. $2 \mathrm{~b}$ shows the most general case of the spring model for structure 2. Each member $i$ is represented as a rigid beam attached at a pin joint, where a torsional spring with spring constant $k_{i}$ models the beam's resistance to deflection. Since the members are fixed-fixed segments, we define $k_{i}=$ $2 \gamma K_{\theta} E I / l_{j}$ [7], where $j=1$ for $i=1$ and $j=2$ otherwise. Here $\gamma=0.85, K_{\theta}=2.68, E$ is Young's modulus, and $I$ is the moment of inertia of the beam. Let $\Delta \theta_{i}$ be the angular displacement of beam $i$ from its undeflected configuration. The moment at each pin joint is $\mathbf{T}_{\mathbf{i}}=-k_{i} \Psi_{i} \hat{\mathbf{k}}$, where $\Psi_{1}=\Delta \theta_{1}, \Psi_{2}=\Delta \theta_{2}-\Delta \theta_{1}, \Psi_{3}=\Delta \theta_{2}-\Delta \theta_{3}, \Psi_{4}=$ $\Delta \theta_{4}-\Delta \theta_{3}, \Psi_{5}=\Delta \theta_{4}-\Delta \theta_{5}$.

We define three cases of the applied force $\mathbf{F}$ in polar coordinates, given in Table I. We use the principle of virtual work to solve for the force-displacement relations. In each
TABLE I

SPRING MODEL PARAMETERS FOR DIFFERENT LOADING CONDITIONS

\begin{tabular}{|c|c|c|c|c|c|c|c|}
\hline & $\mathbf{F}$ & $\theta_{1}$ & $\theta_{2}$ & $\theta_{3}$ & $\theta_{4}$ & $\theta_{5}$ & $\mathcal{I}$ \\
\hline 1 & $F_{r} \mathbf{e}_{r}, \theta=0$ & 0 & $\theta_{q}$ & 0 & $2 \pi-\theta_{q}$ & 0 & $\{2, \ldots, 5\}$ \\
2 & $F_{\theta} \mathbf{e}_{\theta}, \theta \geq 0$ & 0 & $\theta_{q}$ & $\theta_{q}-\frac{\pi}{2}$ & $\theta_{q}+\pi$ & $\theta_{q}-\frac{\pi}{2}$ & $\{2\}$ \\
3 & $F_{\theta} \mathbf{e}_{\theta}, \theta<0$ & $\theta_{q}$ & $\theta_{q}+\frac{\pi}{2}$ & $\theta_{q}$ & $\theta_{q}+\frac{3 \pi}{2}$ & $\theta_{q}$ & $\{1\}$ \\
\hline
\end{tabular}

case, we model the spring as a 1-DOF system with generalized coordinate $\theta_{q}$ and include a subset $\mathcal{I}$ of the torsional springs, corresponding to members that display the most noticeable compliance over the calibration displacement range. We compare the force-displacement models to data from seven calibration trials on the same spring. The calibrated forces are obtained as $x$ and $y$ components and must be transformed into $F_{r}, F_{\theta}$ beforehand. For cases 2 and 3 , the $F_{\theta}$ measurements are interpolated along the circles $\left(x-l_{1}\right)^{2}+y^{2}=\left(l_{3}+l_{5}\right)^{2}$ and $x^{2}+y^{2}=\left(l_{1}+l_{3}+l_{5}\right)^{2}$, respectively.

1) Case 1: Let $r$ denote the spring displacement from its undeformed state. We solve for $F_{r}$ in terms of $\theta_{2}$, which is related to $r$ geometrically:

$$
F_{r}=\frac{\pi / 2-\theta_{2}}{2 l_{2} \sin \left(\theta_{2}\right)} \sum_{i=2}^{5} k_{i}, \quad \theta_{2}=\cos ^{-1}\left(\frac{r}{2 l_{2}}\right) .
$$

Using calibration data, the least squares estimate of $E$ is 1.66 MPa, which is within the typical range for PDMS (360 $\mathrm{kPa}$ to $\sim 3 \mathrm{MPa}$ ) [1]. Fig. 4a shows that equation (1) closely matches the calibration curve.

2) Case 2: We solve for $F_{\theta}$ in terms of $\theta_{2}$. Then from $l_{1}+\left(l_{3}+l_{5}\right) \sin \theta_{2}=z \cos \theta$, where $z=\|\mathbf{z}\|$ :

$$
F_{\theta}=\frac{k_{2}}{z-l_{1} \cos \theta} \cos ^{-1}\left(\frac{z \cos \theta-l_{1}}{l_{3}+l_{5}}\right), \quad \theta \geq 0 .
$$

As Fig. 4b shows, equation (2) matches the averaged calibration data fairly well. Note that the measured $F_{\theta}$ has much greater variability than $F_{r}$.

3) Case 3: We solve for $F_{\theta}$ in terms of $\theta_{1}$, which due to the constraints on the other members is equal to $\theta$ :

$$
F_{\theta}=k_{1} \theta / z, \quad \theta<0 .
$$

Fig. 4b shows that the model underestimates $\left|F_{\theta}\right|$ in this case. A closer fit to the data can be achieved by multiplying $k_{1}$ by 5 , as shown by the light dashed plot.

\section{EXPERIMENTS}

\section{A. Video recording of group retrieval}

Experiments were performed in A. cockerelli's natural Sonoran desert environment in South Mountain Park, Phoenix, Arizona. Ants were induced to retrieve an elastic structure placed approximately one meter from an active nest entrance. The tabs at the ends of the springs were made attractive to the ants by smearing them with fig paste. The structure was placed on a flat wooden board $(50 \mathrm{~cm} \times 40 \mathrm{~cm})$ lined with ivory copy paper. This provided a smooth surface across which the ants could drag the structure, as well as a featureless background to aid in automated video tracking. A high definition video camera (Panasonic HVX200 or Canon HG20) was fixed above 

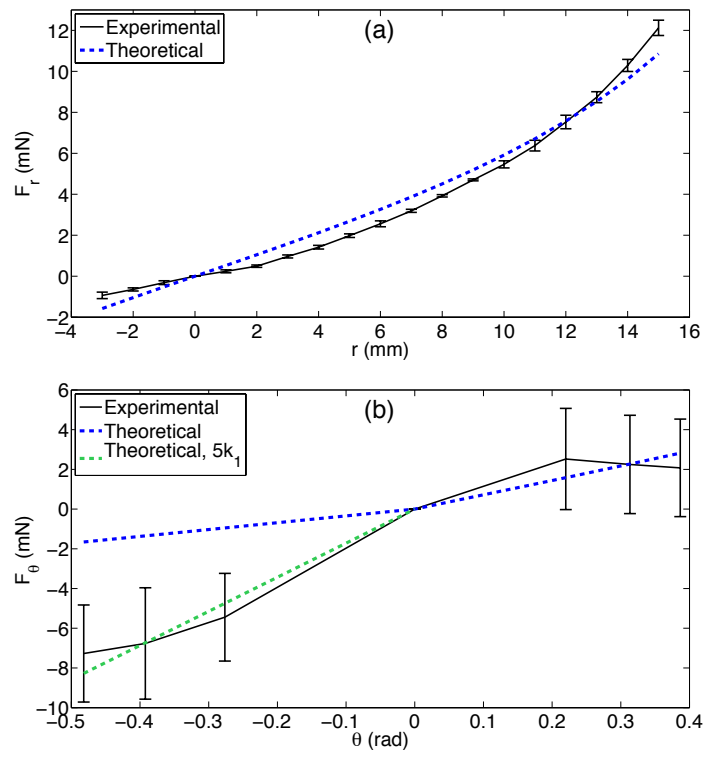

Fig. 4. Experimental and theoretical force-displacement curves: (a) case 1, (b) cases 2 and 3. Measurements are averaged over seven calibration trials. Error bars show standard deviations.

the structure on the vertical post of a copy stand. Its field of view encompassed a region of about $35 \mathrm{~cm} \times 20 \mathrm{~cm}$. A screen blocked direct sunlight from this region, to avoid shadows that could hinder video tracking. Fig baits in the vicinity of the structure were used to attract foragers. Once an ant discovered the structure, she soon initiated recruitment after unsuccessful attempts at moving it herself. A group of four to six ants then cooperated to move the structure toward the nest.

\section{B. Video data image processing}

In order to extract the structure configuration and the spring deformations from the video recordings of the transport, we tracked certain features of the structure, including the load and the tips of the springs. We covered the load with a black circular label marked with a small white circle offset from the center. Using Swistrack [14], a general purpose vision processing software, we used thresholding and blob detection to label and track the load center and the dot. The load orientation was obtained by comparing the relative position of the centers of the dot and the load. The bar on each spring was marked with a red, blue, or green label and was tracked using similar methods. We calculated the deformation of a spring from the position of its label, the load pose, and other geometric quantities.

\section{Results}

1) Mechanics of transport: We found that typical structure inertial forces were on the order of $10^{-4} \mathrm{mN}$, which was negligible compared to the friction force on the structures, $\mu m g=22 \mathrm{mN}$, where $\mu=1.4$ was measured from inclined plane tests with the structures. This indicates that the structures undergo quasi-static motion. The structures were often observed to move by stick-slip, which resulted in noisy data on load speed, $v_{l}$. The average ant force $\left\|\mathbf{F}_{i}\right\|$ was calculated to be $10.5 \pm 5.0 \mathrm{mN}$ (sample size $n=10906$ ); $99.1 \%$ of the samples did not exceed $30.0 \mathrm{mN}$.

2) Cooperative features of transport: Fig. 6 and 8 show snapshots of recorded transports with structures 1 and 2, respectively, as well as the evolution of the load configuration over both trials. Fig. 5 shows the linear and angular speeds of the load over time for the trial in Fig. 6. Fig. 7 displays the sum of the ant interaction forces [10] for the trial in Fig. 8 , each defined as the projection of the difference in forces applied by two ants onto their relative position vector:

$$
\left\|\mathbf{F}_{i j}^{i n t}\right\|=\left(\mathbf{F}_{i}-\mathbf{F}_{j}\right) \cdot\left(\mathbf{r}_{i}-\mathbf{r}_{j}\right) /\left\|\mathbf{r}_{i}-\mathbf{r}_{j}\right\| .
$$

This quantity is a measure of the degree of cooperation between two ants; it is zero when ants are perfectly cooperating.

We see evidence of an initial transport phase of low coordination among the ants followed by a more highly coordinated phase, as has been documented in the literature on group transport in ants (see Section II-A). Fig. 5 shows that structure 1 initially moves slowly on average, and then its speed sharply increases to significantly higher values at $t=68 \mathrm{~s}$. As Fig. 6 reveals, the transition between these phases of speed is initiated by the ants' reorientation of the structure a few degrees counterclockwise. The trial with structure 2 indicates that the phases can also be characterized in terms of the sum of the interaction forces, with the transition occurring as a sizable reduction in this quantity. Fig. 7 shows that this sum decreases to about half its initial value on average after $t=32$ s. From the snapshots in Fig. 8, this increase in cooperation is apparent in the reorientation of the ants to pull with larger force components toward the nest during the second phase.

3) Load speed saturation with increased group size: Fig. 9 shows that three ants are needed to move structure 2, and that when more than five ants participate in transport, there is on average no increase in the load speed. This is due to increased interference between ants during transport with higher populations.

\section{Simulation of Cooperative Manipulation}

In order to simulate realistic motions of the elastic structures that are caused by the ants, we use a planar manipulation model that predicts the motion of an object similar in construction to the structures. In developing the model, we assume quasi-static motion, which was justified experimentally in Section IV-C.1. In addition, we require that friction forces on the springs exceed the spring restorative forces so that a deformed spring cannot, in the absence of ants, cause the entire structure to move.

The simulated object and its associated notation is shown in Fig. 10. The position and orientation of a reference frame $O_{b}$ that is fixed to the load is $(x, y, \theta)$ in an inertial frame $O_{w}$. For each spring $j \in\{1, \ldots, J\}$, we define a frame $R_{j}$ at $\left(x_{R_{j}}, y_{R_{j}}\right)$, a point along the arc where the spring is attached to the load, and a frame $P_{j}$ at $\left(x_{P_{j}}, y_{P_{j}}\right)$, a point at the tip of the spring. The orientation of $R_{j}$ in frame $O_{w}$ is denoted by $\theta_{R_{j}}$, and 

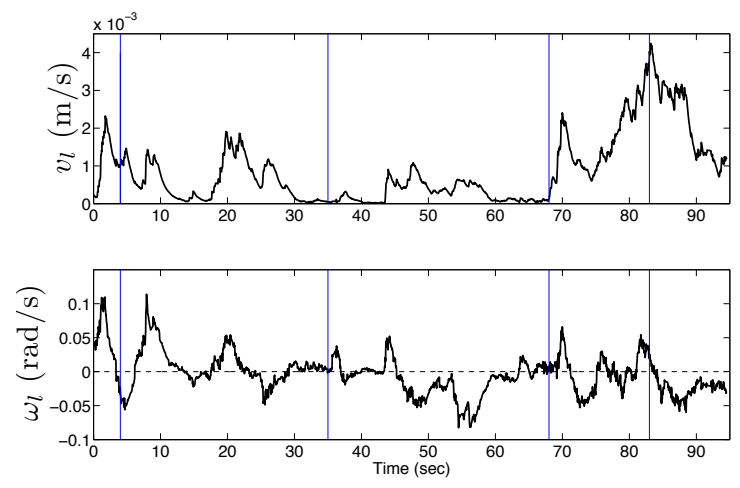

Fig. 5. Linear and angular load speeds over time for the trial in Fig. 6. Vertical lines indicate the times of the snapshots.
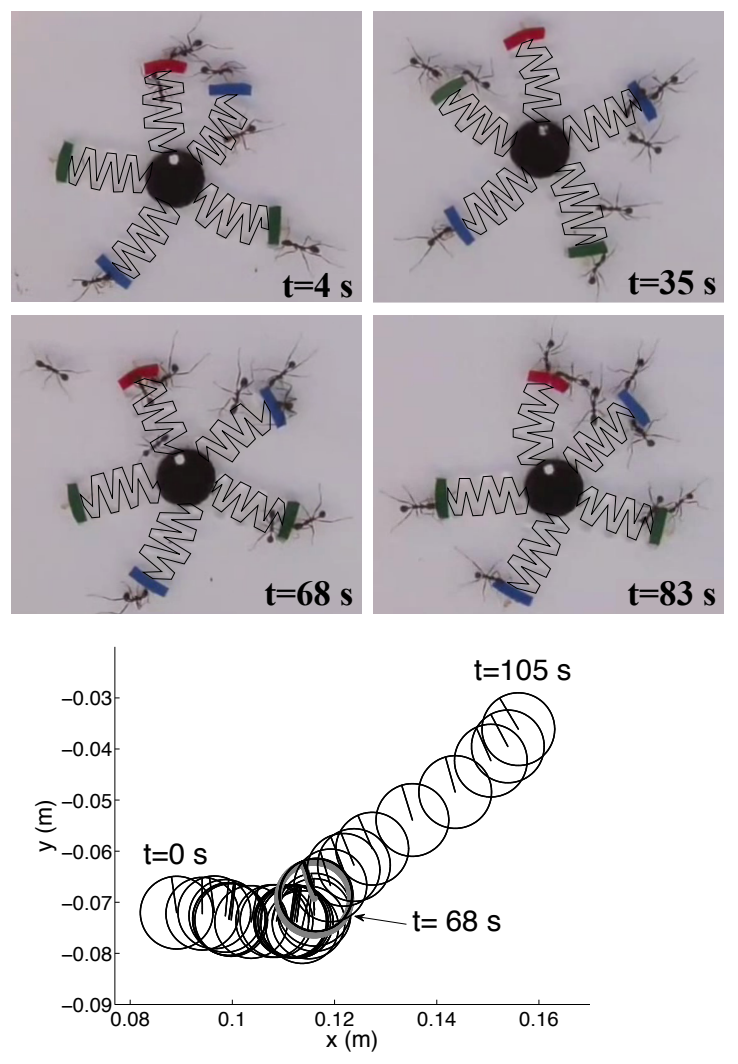

Fig. 6. Snapshots of ant transport with structure 1 (top); load position and orientation (bottom). The structure springs are outlined for clarity. The thick gray circle in the bottom plot signifies the load at $t=68 \mathrm{~s}$.

the orientation of $P_{j}$ is $\theta_{P_{j}}$, which is set to the orientation of frame $O_{w}$. We define a homogeneous transformation matrix

$$
\mathbf{A}(x, y, \theta)=\left[\begin{array}{ccc}
\cos \theta & -\sin \theta & x \\
\sin \theta & \cos \theta & y \\
0 & 0 & 1
\end{array}\right] .
$$

Then we can represent the configurations just described as ${ }^{O} \mathbf{A}_{O_{b}} \equiv \mathbf{A}(x, y, \theta),{ }^{O_{w}} \mathbf{A}_{R_{j}} \equiv \mathbf{A}\left(x_{R_{j}}, y_{R_{j}}, \theta_{R_{j}}\right)$, and ${ }^{{ }_{w}} \mathbf{A}_{P_{j}} \equiv \mathbf{A}\left(x_{P_{j}}, y_{P_{j}}, \theta_{P_{j}}\right)$.

Using the notation in [18], we define the load twist in the

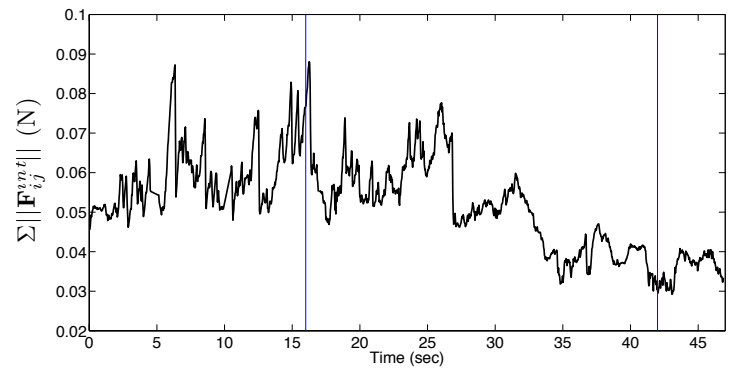

Fig. 7. Sum of interaction forces over time for the trial in Fig. 8. Vertical lines indicate the times of the snapshots.
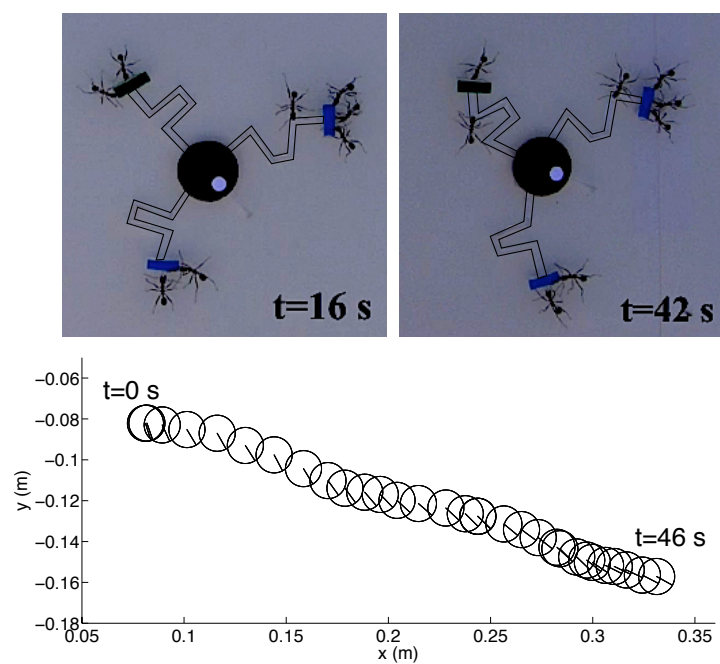

Fig. 8. Snapshots of ant transport with structure 2 (top); load position and orientation (bottom). The structure springs are outlined for clarity.

inertial frame as

$$
\hat{\xi}_{\text {load }}={ }^{O_{w}} \mathbf{A}_{O_{b}}^{-1}{ }^{O_{w}} \dot{\mathbf{A}}_{O_{b}}
$$

or in terms of the vector

$$
\xi_{\text {load }}=\left[\begin{array}{lll}
\dot{x} & \dot{y} & \dot{\theta}
\end{array}\right]^{T} .
$$

Similarly, we can define the displacement of frame $P_{j}$ from its undeformed configuration in the frame $R_{j}$ :

$$
\begin{gathered}
{ }^{R_{j}} \mathbf{A}_{P_{j}}={ }^{O_{w}} \mathbf{A}_{R_{j}}^{-1} O_{w} \mathbf{A}_{P_{j}}, \\
\hat{\xi}_{s p, j}={ }^{R_{j}} \mathbf{A}_{P_{j}}^{-1} R_{j} \dot{\mathbf{A}}_{P_{j}} .
\end{gathered}
$$

We model each spring $j$ as a composition of an extension spring and a rotary spring, resulting in a wrench $\mathbf{w}_{j}$ in the frame $R_{j}$ with the following form:

$$
\begin{aligned}
& \mathbf{F}_{j}=-\frac{\kappa_{\tau} \theta_{s p, j}}{l}\left[\begin{array}{c}
-\sin \left(\theta_{P_{j}}+\theta\right) \\
\cos \left(\theta_{P_{j}}+\theta\right)
\end{array}\right]-\kappa_{\rho}\left(l-l_{o}\right)\left[\begin{array}{c}
\cos \left(\theta_{P_{j}}+\theta\right) \\
\sin \left(\theta_{P_{j}}+\theta\right)
\end{array}\right] \\
& \mathbf{w}_{j}=\left[\begin{array}{c}
\mathbf{F}_{j} \\
x_{s p, j} F_{j, y}-y_{s p, j} F_{j, x}
\end{array}\right],
\end{aligned}
$$

where $l=\left(x_{s p, j}^{2}+y_{s p, j}^{2}\right)^{1 / 2}$ is the deformed length of the spring, $l_{0}$ is the free length of the spring, $\kappa_{\tau}$ is the rotational torque constant, and $\kappa_{\rho}$ is the extension spring constant. 


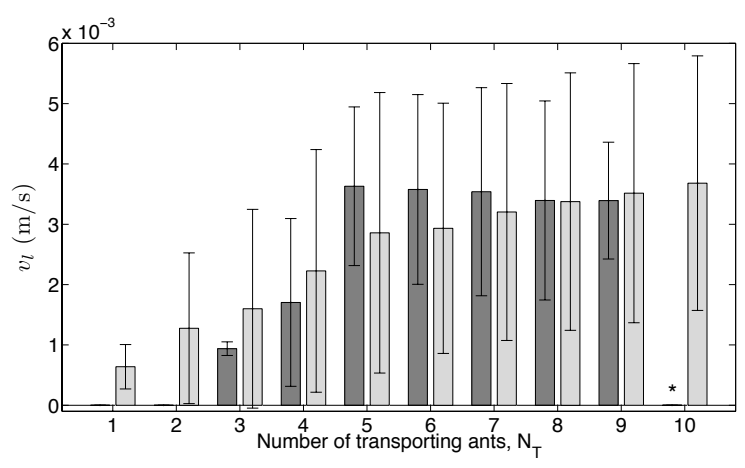

Fig. 9. Average load speed vs. transport group size in the experimental trials with structure 2 (dark gray bars) and simulation trials (light gray bars), which are described in Section VII. Error bars show standard deviations. Experiment sample sizes for $N_{T}=4-9$ are $n=561,453,4154,2448,1866,182$; data for $N_{T}=1-3$ were obtained manually, and $*$ indicates that no data were available. Simulation sample sizes for $N_{T}=1-10$ are $n=1186594$, 19971, 50522, 36362, 35459, 4956, 17700, 38777, 46289, 19234.

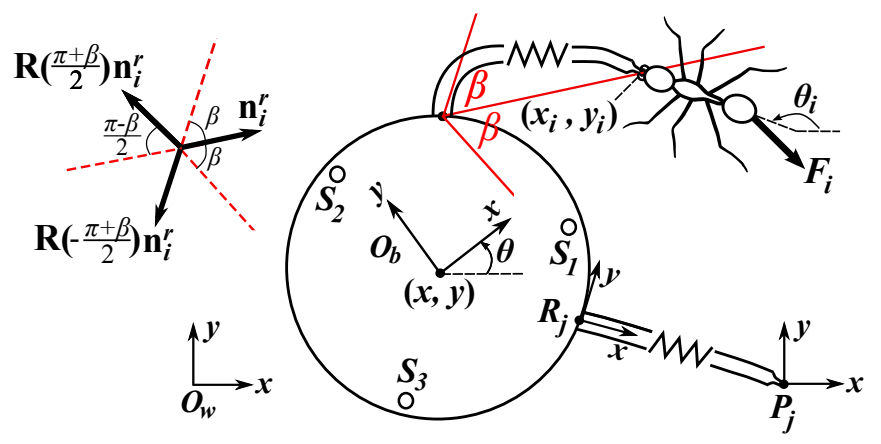

Fig. 10. Notation used in Sections V and VI for a simulated elastic structure with a transporting ant (two springs are shown).

To model friction, we use a finite support point approach similar to the one in [3]. We assume that the object is supported by three support points $S_{i}, i=1,2,3$, whose coordinates are $\left(x_{S, i}, y_{S, i}\right)$ in frame $O_{b}$. The friction forces $\lambda_{t}$ comprise an unknown $6 \times 1$ vector:

$$
\lambda_{t}=\left[\begin{array}{lll}
\lambda_{t, 1} & \lambda_{t, 2} & \lambda_{t, 3}
\end{array}\right]^{T}, \quad \lambda_{t, i}=\left[\begin{array}{ll}
\lambda_{t, i, x} & \lambda_{t, i, y}
\end{array}\right] .
$$

The friction cone at the $i$ th support point with a known normal force $\lambda_{n, i}$ is denoted by $\mathcal{F C}_{i}$ and is defined by Coulomb friction: $0 \leq\left\|\lambda_{t, i}\right\| \leq \mu \lambda_{n, i}$. We can write the $2 \times 1$ velocity vector of the support point for a load twist as:

$$
v_{t, i}=\left[\begin{array}{ccc}
1 & 0 & -y_{S, i} \\
0 & 1 & x_{S, i}
\end{array}\right] \quad \xi_{\text {load }} \equiv \mathbf{B}_{i} \xi_{\text {load }} .
$$

From Coulomb's law, the friction forces are equal to $\mu \lambda_{n, i}$ and are opposite to the direction of slip, except if the slip is zero, in which case the magnitude is indeterminate. This can be written explicitly as:

$$
\lambda_{t, i}\left(\xi_{\text {load }}\right) \in \underset{\lambda_{i} \in \mathcal{F} \mathcal{C}_{i}}{\arg \min } v_{t, i}\left(\xi_{\text {load }}\right)^{T} \lambda_{i}
$$

or in aggregate form,

$$
\lambda_{t}\left(\xi_{\text {load }}\right) \in \underset{\lambda_{i} \in \mathcal{F} \mathcal{C}_{i}}{\arg \min } \xi_{\text {load }}^{T} \mathbf{B}^{T} \lambda
$$

where $\mathbf{B}^{T}=\left[\begin{array}{lll}\mathbf{B}_{1}^{T} & \mathbf{B}_{2}^{T} & \mathbf{B}_{3}^{T}\end{array}\right]$.

We can determine the motion of the load by solving the following max-min problem,

$$
\underset{\xi_{\text {load }} \in \Sigma}{\operatorname{maximize}} \underset{\lambda_{t, i} \in \mathcal{F C}_{i}}{\operatorname{minimize}} L\left(\xi, \lambda_{t}\right)
$$

where $L$ is the instantaneous power from the friction forces and the wrenches caused by the spring deformations:

$$
L\left(\xi, \lambda_{t}\right)=\xi_{\text {load }}^{T} \mathbf{B}^{T} \lambda_{t}+\sum_{j=1}^{J} \xi_{s p, j}^{T} \mathbf{w}_{j} .
$$

\section{BEHAVIORAL MODEL}

We developed a model of the ant transport behavior from qualitative observations of the videos of transport trials. The transport strategy is represented as a hybrid system with probabilistic transitions between task modes. Fig. 10 illustrates the notation that we use in describing the model.

We consider a population of $N$ ants, each represented as a point-mass agent governed by a kinematic model,

$$
\dot{\mathbf{q}}_{i}=\mathbf{u}_{i}
$$

where $\mathbf{q}_{i}=\left[\begin{array}{ll}\mathbf{r}_{i} & \theta_{i}\end{array}\right]^{T}=\left[\begin{array}{lll}x_{i} & y_{i} & \theta_{i}\end{array}\right]^{T}$ denotes the position of a point associated with ant $i$ and the ant's orientation, both in the inertial frame, and $\mathbf{u}_{i}=\left[\begin{array}{lll}u_{i}^{x} & u_{i}^{y} & u_{i}^{\theta}\end{array}\right]^{T}$ is the vector of control inputs.

We model the ants as switching between two behavioral modes, each corresponding to a different $\mathbf{u}_{i}$. In the behavior Search for grasp point, an ant $i$ moves toward the structure in search of a protrusion to latch onto with its mandibles. If the outermost tip of a spring enters the sensing range of the ant and the spring is not already occupied by another ant, the ant latches onto the spring with probability $p_{1}$. Once attached, the ant is in the Transport mode and pulls on the spring in a manner that directs the structure toward its estimate of the nest. The ant reverts to mode Search for grasp point with a probability per time step $p_{2, i}(t)$, which is defined as a sigmoid function of the magnitude of the force $\mathbf{F}_{i}(t)$ that the ant applies to the spring at time $t$ :

$$
p_{2, i}(t)=\left(1+e^{-\zeta\left(\left\|\mathbf{F}_{i}(t)\right\|-0.5 F_{m}\right)}\right)^{-1},
$$

where $F_{m}$ is a force close to the maximum force that the ant can apply and $\zeta>1$. We chose a sigmoid function to reflect the ants' tendencies to release the spring more often at large deflections and to occasionally lose their grip or lose interest even at low deflections. The ants stop moving when $\left\|\mathbf{r}-\mathbf{r}_{n}\right\| \leq \rho$, where $\mathbf{r}=\left[\begin{array}{ll}x & y\end{array}\right]^{T}$ and $\mathbf{r}_{n}=\left[\begin{array}{ll}x_{n} & y_{n}\end{array}\right]^{T}$, the position of the nest, for some small constant $\rho$.

\section{A. Search for grasp point}

We define $v_{i}$ and $\omega_{i}$ as the forward linear and angular speeds, respectively, of ant $i$. An ant's estimates of the structure position $(x, y)$, given by $(\hat{x}, \hat{y})$, are defined as values of the random variables $\hat{X} \sim \mathcal{N}\left(x, \sigma^{2}\right)$ and $\hat{Y} \sim \mathcal{N}\left(y, \sigma^{2}\right)$, where $\sigma^{2}$ is a specified variance. These values are updated at each time step. The ant's desired orientation $\theta_{i}^{d}$ is defined as 
the angle of the vector from $\left(x_{i}, y_{i}\right)$ to $(\hat{x}, \hat{y})$. A proportional controller is used to steer the ant's orientation to $\theta_{i}^{d}$ :

$$
\omega_{i}=k e_{i}^{\theta}, \quad e_{i}^{\theta}=\left(\theta_{i}^{d}-\theta_{i}\right) \in[-\pi, \pi] .
$$

A feedback linearization scheme is used to relate $v_{i}$ and $\omega_{i}$ to the linear velocities of $\left(x_{i}, y_{i}\right)$. Hence the control inputs are

$$
\left[\begin{array}{l}
u_{i}^{x} \\
u_{i}^{y} \\
u_{i}^{\theta}
\end{array}\right]=\left[\begin{array}{cc}
\cos \theta_{i} & -r \sin \theta_{i} \\
\sin \theta_{i} & r \cos \theta_{i} \\
0 & 1
\end{array}\right]\left[\begin{array}{l}
v_{i} \\
\omega_{i}
\end{array}\right],
$$

where $r$ is a small offset distance along the longitudinal axis of the ant.

\section{B. Transport}

An ant $i$ in this mode is grasping the tip of spring $j$. The ant pulls on its attachment point, whose coordinates are given by $\left(x_{i}, y_{i}\right)=\left(x_{P_{j}}, y_{P_{j}}\right)$, with a force $\mathbf{F}_{i}$. Let $\mathbf{n}_{i}^{f}$ be the unit vector in the direction of this force. Also define $\mathbf{n}_{i}^{n}$ as the unit vector from $\left(x_{i}, y_{i}\right)$ toward the nest coordinates, $\left(x_{n}, y_{n}\right)$, and $\mathbf{n}_{i}^{r}$ as the unit vector from $\left(x_{R_{j}}, y_{R_{j}}\right)$, to $\left(x_{i}, y_{i}\right)$. The angles of these vectors in the inertial frame are $\theta_{i}+\pi, \theta_{i}^{n}$, and $\theta_{i}^{r}$, respectively. To navigate to their nest (i.e., determine $\theta_{i}^{n}$ ), ants use visual landmarks and path integration with celestial cues as a compass [25].

We observed that an ant grasping a spring will attempt to rotate the load into an orientation in which the ant can pull it while walking backward toward the nest. To emulate this behavior, which has also been noted in [23], we specify that at each time $t$, the ant can begin to reorient itself to any angle between $\theta_{i}^{\min }(t)=\theta_{i}^{r}(t)-\beta$ and $\theta_{i}^{\max }(t)=\theta_{i}^{r}(t)+\beta$, where $\beta \leq \pi / 2$. Similarly to equation (19), a proportional controller steers $\theta_{i}$ :

$$
\omega_{i}=k e_{i}^{\theta}, \quad e_{i}^{\theta}=\left(\theta_{i}^{d}-\left(\theta_{i}+\pi\right)\right) \in[-\pi, \pi] .
$$

Fig. 10 illustrates a division of the plane into three regions that are bounded by light dashed lines; in the diagram, $\mathbf{R}(\theta)$ is the $2 \mathrm{D}$ rotation matrix. The desired orientation $\theta_{i}^{d}$ at a given time depends on which of the regions contains $\mathbf{n}_{i}^{n}$ :

$$
\theta_{i}^{d}=\left\{\begin{array}{lll}
\theta_{i}^{n} & \text { if } & \mathbf{n}_{i}^{r} \cdot \mathbf{n}_{i}^{n} \geq \cos \beta \\
\theta_{i}^{\text {max }} & \text { if } & \mathbf{R}\left(\frac{\pi+\beta}{2}\right) \mathbf{n}_{i}^{r} \cdot \mathbf{n}_{i}^{n} \geq \cos \frac{\pi-\beta}{2} \\
\theta_{i}^{\text {min }} & \text { if } & \mathbf{R}\left(-\frac{\pi+\beta}{2}\right) \mathbf{n}_{i}^{r} \cdot \mathbf{n}_{i}^{n} \geq \cos \frac{\pi-\beta}{2}
\end{array}\right.
$$

The control inputs are defined as follows:

$$
\mathbf{u}_{i}=\left[\begin{array}{lll}
-v_{i} \cos \theta_{i} & -v_{i} \sin \theta_{i} \omega_{i}
\end{array}\right]^{T} .
$$

\section{ViI. Collective Transport Simulation}

We simulated transport scenarios with different numbers of ants and springs on the elastic structure. The configurations of the structure and the ants are updated at each time step using the dynamic manipulation model in Section $\mathrm{V}$ and the behavioral model in Section VI. The simulated structure has the dimensions and weight of structure 2, with spring constants $\kappa_{\tau}=4.52 \times 10^{-4} \frac{\mathrm{Nm}}{\mathrm{rad}}$ and $\kappa_{\rho}=0.664 \frac{\mathrm{N}}{\mathrm{m}}$ obtained from the spring model in Section III-D. We selected $p_{1}=0.2$ and $\beta=$ $60^{\circ}$ to emulate the observed ant behavior and $F_{m}=20 \mathrm{mN}$ to achieve maximum ant forces near the experimental value of $\sim 30 \mathrm{mN}$ (see Section IV-C.1). We also set $k=10 \mathrm{~s}^{-1}$, $r=2 \mathrm{~mm}, \zeta=100$, and $\sigma^{2}=0.01$. The ants initially have uniformly randomly distributed positions and orientations. All ants start in Search for grasp point mode, in which they move at $v_{i}=0.08 \mathrm{~m} / \mathrm{s}$, which was estimated from video data. The nest is located at $(0.2 \mathrm{~m},-0.17 \mathrm{~m})$.

Fig. 12 shows snapshots of a simulation with four ants and four springs and the resulting load trajectory and orientation, and Fig. 11 gives the corresponding load speeds and sum of interaction forces over time. We also conducted trials with one ant/one spring, five ants/ten springs, and ten ants/ten springs. In the simulations, the average ant force $\left\|\mathbf{F}_{i}\right\|$ was $5.0 \pm 5.1$ $\mathrm{mN}$ and the maximum force was $36.5 \mathrm{mN}$ (sample size $n=$ 520057), which are comparable to the experimental values. The interaction force sum in Fig. 11 varies over a similar range to the sum in Fig. 7. Fig. 9 shows that the simulations produced average $v_{l}$ that were fairly close to the observed $v_{l}$ for $N_{T}=3-9$. In addition, the figure illustrates that the simulations reproduce the effect of diminishing increases in average $v_{l}$ with larger transport populations, although the speed may not have saturated yet at $N_{T}=10$.

The simulations replicate not only the successful transport of the structure to the nest, as shown by the trajectory in Fig. 12 , but also the distinctive property of a phase transition to a higher degree of coordination among individuals. The speed $v_{l}$ in Fig. 11 displays a similar trend to the experimental $v_{l}$ in Fig. 5: a phase of low average speed with relatively small peaks is followed by a phase of increased speed. In the simulation, this transition is also marked by increased angular rotation of the load, as shown by the plot of $\omega_{l}$. While the sum of the interaction forces in Fig. 11 does not obviously signal the phase change as it does in Fig. 7, it displays a sharp reduction around $t=83 \mathrm{~s}$ that coincides with the jump in $v_{l}$. The snapshots in Fig. 12 show that the the transporting ants are arranged in a less cooperative pose in the first phase than in the second, as occurs in the snapshots of the experiments.

\section{CONCLUSION}

We have presented a study of the mechanics of cooperative prey retrieval in A. cockerelli ants based on experimental trials with vision-based force sensors. The data show that this distributed transport system is characterized by an initial disordered phase that transitions to a coordinated phase of increased load speed and a higher degree of cooperation among the transporters, as well as a plateau in the progress of the task after a threshold population is reached. We developed a quasi-static dynamic manipulation model and an ant behavioral model based on observations and incorporated these models into a simulation that replicates the experimental phenomena.

In future work, we would like to conduct further experiments to extract the ants' changing roles, examine the mechanics of lifting during transport, and investigate the role of sustained interaction forces in helping to avoid obstacles and overcome deadlocks. Another objective is the implementation of the ant-like cooperative transport strategies on the robots in 

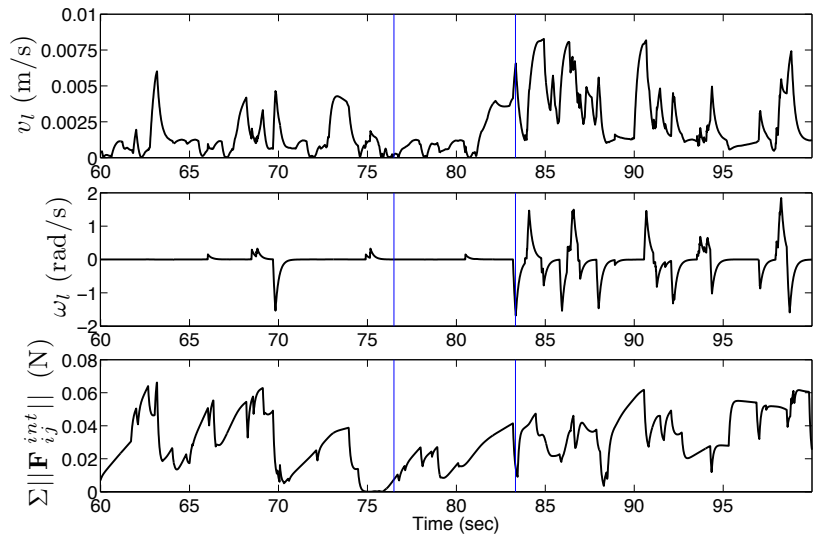

Fig. 11. Linear and angular load speeds and sum of interaction forces over time for the simulated trial in Fig. 12. Vertical lines indicate the times of the snapshots.
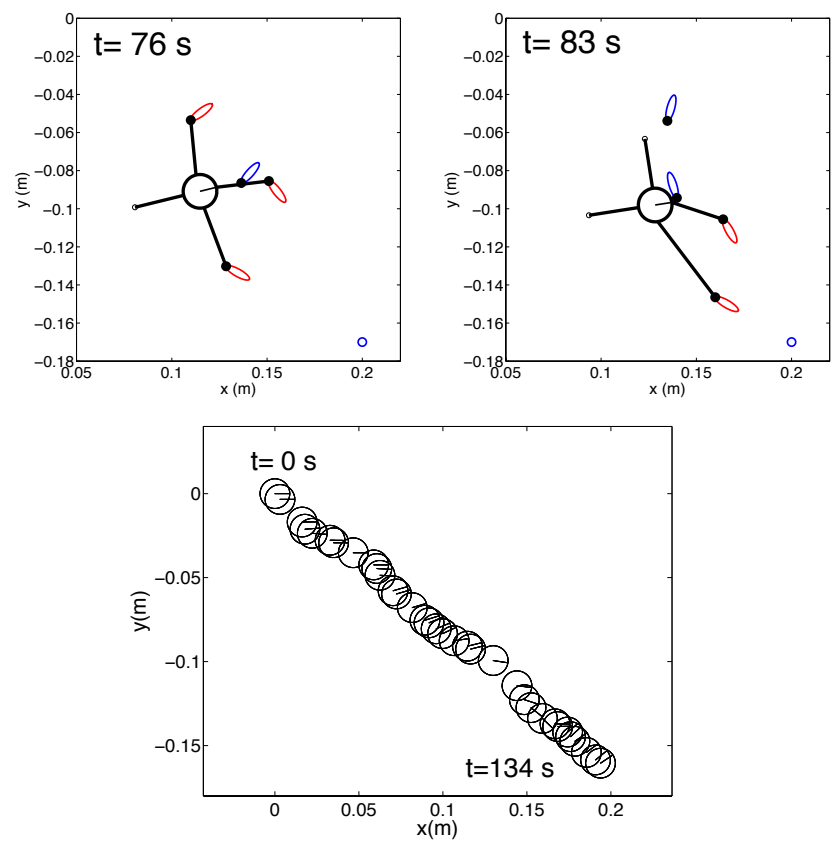

Fig. 12. Snapshots of simulated ant transport (top two plots); load position and orientation (bottom). In the top two plots, ants that are attached to the tips of the springs are in Transport mode, the other ants are in Search for grasp point mode, and the nest is indicated by the circle at $(0.2 \mathrm{~m},-0.17 \mathrm{~m})$.

Fig. 1. The dynamic manipulation model has been adapted to these robots and validated in [11]. The implementation will require decentralized approaches for locating attachment points on an object, such as through a vision-based method; grasping these points firmly; agreeing on the target destination through a consensus algorithm if it is not preprogrammed; and navigating toward this location while avoiding obstacles and other robots. While ants can sidestep, the robots are nonholonomic and so they cannot imitate these maneuvers, which must be taken into account in their motion control.

\section{ACKNOWLEDGMENTS}

We gratefully acknowledge support from NSF IIS-0427313, ARO Grant W911NF-05-1-0219, and ONR Grants N0001407-1-0829 and N00014-08-1-0696.

\section{REFERENCES}

[1] D. Cappelleri, G. Piazza, and V. Kumar. Two-dimensional, vision-based $\mu \mathrm{N}$ force sensor for microrobotics. In Proc. Int'l. Conf. on Robotics and Automation (ICRA), Kobe, Japan, 2009.

[2] R. Chauvin. Le transport de proies chez les fourmis. Y-a-t-il entr'aide? Behaviour, 2(4):249-256, 1950.

[3] P. Cheng, J. Fink, V. Kumar, and J. S. Pang. Cooperative towing with multiple robots. ASME J. Mechanisms and Robotics, 1(1), 2009.

[4] J. Fink, N. Michael, and V. Kumar. Composition of vector fields for multi-robot manipulation via caging. In Robotics: Science and Systems (RSS), 2007

[5] B. Holldobler, R. C. Stanton, and H. Markl. Recruitment and foodretrieving behavior in Novomessor (Formicidae, Hymenoptera: I. Chemical signals). Behav. Ecol. Sociobiol., 4:163-181, 1978.

[6] B. Holldobler and E. O. Wilson. The Ants. Harvard University Press, Cambridge, MA, 1990.

[7] L. Howell. Compliant Mechanisms. John Wiley \& Sons, Inc., New York, 2001.

[8] O. Khatib, K. Yokoi, K. Chang, D. Ruspini, R. Holmberg, and A. Casal. Coordination and decentralized cooperation of multiple mobile manipulators. J. of Robotic Systems, 13(11):755-764, 1996.

[9] C. R. Kube and E. Bonabeau. Cooperative transport by ants and robots. Robotics and Autonomous Systems, 30:85-101, 2000.

[10] V. Kumar and K. Waldron. Force distribution in closed kinematic chains. IEEE J. Robotics and Automation, 4(6):657-664, 1988.

[11] Q. Lindsey, M. Shomin, and V. Kumar. Cooperative quasi-static planar manipulation with multiple robots. In ASME IDETC/CIE, 2010.

[12] Arnaud Lioni. Ch. VII: Le transport collectif chez Oecophylla longinoda. From: Auto-assemblage et transport collectif chez Oecophylla. Unpublished. PhD thesis, Univ. Libre de Bruxelles, Belgium, 2000.

[13] Y. Liu, S. Arimoto, V. Parra-Vega, and K. Kitagaki. Decentralized adaptive control of multiple manipulators in co-operations. Int'l. J. of Control, 67(5):649-673, 1997.

[14] T. Lochmatter, P. Roduit, C. Cianci, N. Correll, J. Jacot, and A. Martinoli. SwisTrack - A Flexible Open Source Tracking Software for MultiAgent Systems. In Proc. IEEE/RSJ Int'l. Conf. on Intelligent Robots and Systems (IROS), pages 4004-4010, 2008.

[15] N. Michael, J. Fink, and V. Kumar. Experimental testbed for large multirobot teams: verification and validation. IEEE Robotics and Automation Magazine, 15(1):53-61, 2008.

[16] M. W. Moffett. Cooperative food transport by an Asiatic ant. National Geographic Research, 4(3):386-394, 1988.

[17] G. Montemayor and J. Wen. Decentralized collaborative load transport by multiple robots. In Proc. Int'l. Conf. on Robotics and Automation (ICRA), pages 372-377, 2005.

[18] R. Murray, Z. Li, and S. Sastry. A Mathematical Introduction to Robotic Manipulation. CRC Press, Florida, 1994.

[19] G. A. S. Pereira, V. Kumar, and M. F. M. Campos. Decentralized algorithms for multi-robot manipulation via caging. Int'l. Journal of Robotics Research, 23:783-795, 2002.

[20] H. Su and V. Krovi. Decentralized dynamic control of a nonholonomic mobile manipulator collective: a simulation study. In Proc. ASME Dynamic Systems and Control Conf. (DSCC), Ann Arbor, MI, 2008.

[21] J. H. Sudd. The transport of prey by an ant, Pheidole crassinoda. Behaviour, 16:295-308, 1960.

[22] J. H. Sudd. How insects work in groups. Discovery, 24(6):15-19, 1963.

[23] J. H. Sudd. The transport of prey by ants. Behaviour, 25:234-271, 1965.

[24] D. Sun and J. K. Mills. Manipulating rigid payloads with multiple robots using compliant grippers. IEEE/ASME Trans. on Mechatronics, $7(1): 23-34,2002$.

[25] R. Wehner. The architecture of the desert ant's navigational toolkit (Hymenoptera: Formicidae). Myrmecological News, 12:85-96, 2009.

[26] Y. Zhao, C. C. Lim, D. B. Sawyer, L. Ronglih, and X. Zhang. Cellular force measurements using single-spaced polymeric microstructures: isolating cells from base substrate. J. Micromech. Microeng., 15:16491656, 2005. 